\title{
Research on the Project Selection Path of Counseling Graduate Students' Entrepreneurship Plan Driven by TRIZ Resource Analysis
}

\author{
Jing Guo ${ }^{1,}$, , Xiaolin Sun ${ }^{2}$ \\ ${ }^{1}$ Tianjin University of Finance and Economics, Centre for innovation and entrepreneurship, Tianjin, 300222, China \\ ${ }^{2}$ Tianjin University of Finance and Economics, International Business School, Tianjin, 300222, China \\ *Corresponding author: Jing Guo (Email: hebutguojing@126.com)
}

\begin{abstract}
Taking the entrepreneurship plan project as the carrier to cultivate college students' innovative and entrepreneurial thinking and ability is core content of college' entrepreneurship education in China. Facing the background of the dynamic development of policy, economy, society and technology, there is an urgent need to break the discipline limitations and scientific methods to identify the entrepreneurial opportunities. Although the existing entrepreneurial management theory provides a basis for the source of entrepreneurial opportunities, the counseling practice shows that college students still have difficulties to break through the thinking constraints and innovation bottlenecks in the selection process of entrepreneurial plan project. Based on the perspective of effectual logic, this study introduces TRIZ "9 windows" resource analysis tool, combines it with PEST model, designs the selection path of entrepreneurship plan project, and applies it to the process of tutoring college students' Entrepreneurship plan project. Through the counseling practice of College Students' innovation and Entrepreneurship Program in 2021, the effectiveness of the design path of this study is tested.
\end{abstract}

Keywords: College students, Entrepreneurship plan, TRIZ, 9 windows, Counseling path.

\section{Introduction}

In recent years, the development of innovation and entrepreneurship education driven by project guidance has attracted extensive attention in China [1]. Taking the project selection and implementation plan as the carrier, improving the ability of college students to identify opportunities in an uncertain environment, and finally cultivating college students' innovative and entrepreneurial thinking is the guidance of entrepreneurship education.

Combined with the teaching of entrepreneurship courses and the experience of guiding students' entrepreneurship projects, it is found that despite existing entrepreneurship management research provides some methods for identifying entrepreneurship opportunities [2] [3], such as new vision survey, system analysis, problem analysis, customer suggestions, creating needs etc., there are still some problems for undergraduates to design the project plan, such as the lack of innovation or practical value. The reason is that in the process of identifying opportunities, most student teams mainly focus on their own resources, look for pain points and identify opportunities from the perspective of static analysis, and they do not systematically find resources. Therefore, how to break the bottleneck of knowledge, information and technology, and integrate relevant resources based on the core competitive advantages to tap potential entrepreneurial opportunities needs to be guided by other scientific methods.

Driven by the effectual logic, this study introduces the "9 windows" method in TRIZ and PEST model, realizes the comprehensive analysis of system evolution and its causes through the time axis and system axis, puts forward the entrepreneurial opportunity identification method to break the thinking inertia, and constructs the selection path of counseling entrepreneurial projects. It realizes dynamic analysis of resources in the process of college students' entrepreneurship plan project selection, provide reference for college students' entrepreneurship plan project selection and instructors' guidance for entrepreneurship plan projects, and supplement the existing entrepreneurship management research theory.

\section{Related Work}

The existing methods of innovation and entrepreneurship project counseling are mainly proposed from the perspective of suggested strategies [4] [5]. For example, Yang (2013) [4] proposed that the college entrepreneurship project guidance and guidance model includes the following three points: starting from the foundation and striving to cultivate students' innovation ability; improving the faculty of entrepreneurship counseling in colleges and universities; Enriching the campus entrepreneurship atmosphere and strengthening the construction of entrepreneurship counseling courses. Liu (2018) [5] explored how to carry out personalized and differentiated counseling for entrepreneurship projects for higher vocational colleges by building a new entrepreneurial mentor team, creating a new entrepreneurial service platform and exploring a new entrepreneurial counseling model. The existing research provides guidance for the counseling and incubation of college students' innovation and entrepreneurship projects. However, they were proposed from macro perspective, and they did not provide specific methods, so that the counseling process still depends more on experience and lacks guiding significance for the improvement of counseling and incubation ability.

The guidance and incubation of college students' entrepreneurship plan projects must reflect college students' innovative ability and entrepreneurial thinking, which is also the core of innovation and entrepreneurship education in universities. Facing the construction of new engineering and new liberal arts, promoting the organic integration of 
interdisciplinary, the adoption of scientific methods to promote the incubation of innovation and entrepreneurship projects is an important breakthrough. The reasons include the following two aspects: firstly, in the process of choosing entrepreneurship training projects, it is difficult for undergraduate students to produce innovative ideas and incubate them into entrepreneurship projects. Therefore, scientific methods are needed to break through the shackles of thinking inertia; Secondly, the knowledge limitation of the discipline is a double-edged sword. In the process of strengthening professional knowledge, it is also easy to fall into certain industry constraints. Under the current background, entrepreneurial projects require high intensity innovation, and the analysis of entrepreneurial opportunities usually involves more than one industry. Therefore, the introduction of scientific methods is needed to help entrepreneurs tap opportunities that are easy to be ignored.

\section{Counseling Path of Entrepreneurship Plan Project for College Students}

\subsection{Identification of Entrepreneurship Opportunities Based on 9 Windows and PEST Model}

In the research field of TRIZ (Theory of inventive problem solving), resources are defined as the sum of all things inside and outside the system that can be applied to the greatest potential [7]. "9 window method" is one of the important tools for analyzing resources in TRIZ. It makes a joint dynamic analysis of internal and external resources and their past and future states by constructing time axis and system axis. At present, this method is mainly used in the process of product innovation.

PEST model is to analyze the macro environment from political, economic, social and technological aspects. The macro environment refers to various macro factors affecting all industries and enterprises [2]. This study introduces the "9 windows" method, combined with PEST analysis, regards the current project as a system, analyzes the products and services, business model of the current system, as well as the technological evolution and causes of subsystems, and constructs the process of entrepreneurial opportunity identification. The specific steps are as follows:

Step 1: Based on the current state of the timeline, first analyze the "current system and subsystem", analyze the market positioning of the current system (project), the products and services provided (including function, performance, structure or materials), technical means, marketing mode and business model, etc.

Step 2: Analyze the "super system" of the current system. According to pest model, the super system resources are analyzed from the four dimensions of policy, economy, society, culture and technology, such as relevant policies of the industry to which the system belongs, economic situation, changes in the proportion of population age or demand, advanced technology, etc.

Step 3: Observe backwards along the time axis and analyze the "past tense of current system and subsystem" and "past tense of super system", that is, analyze the market positioning, products and services provided, technical means, marketing mode and business model of previous projects; compare the changes and reasons of "subsystem of current system" relative to "past tense of subsystem of current system". The main problems are as follows: (1) What subsystems have existed from the past to the present? (2) Have these subsystems changed? (3) What causes these subsystems to change? (4) What new systems have emerged? (5) What's new? (6) Why do these new features appear?

Step 4: According to the reasons found in step 3, combined with the "super system" resources, analyze whether these reasons can continue to promote the evolution of subsystems. In addition, analyze whether there are changes in super system resources, which can promote the evolution of subsystems, and even develop new systems to build "subsystem future tense" and "system future tense".

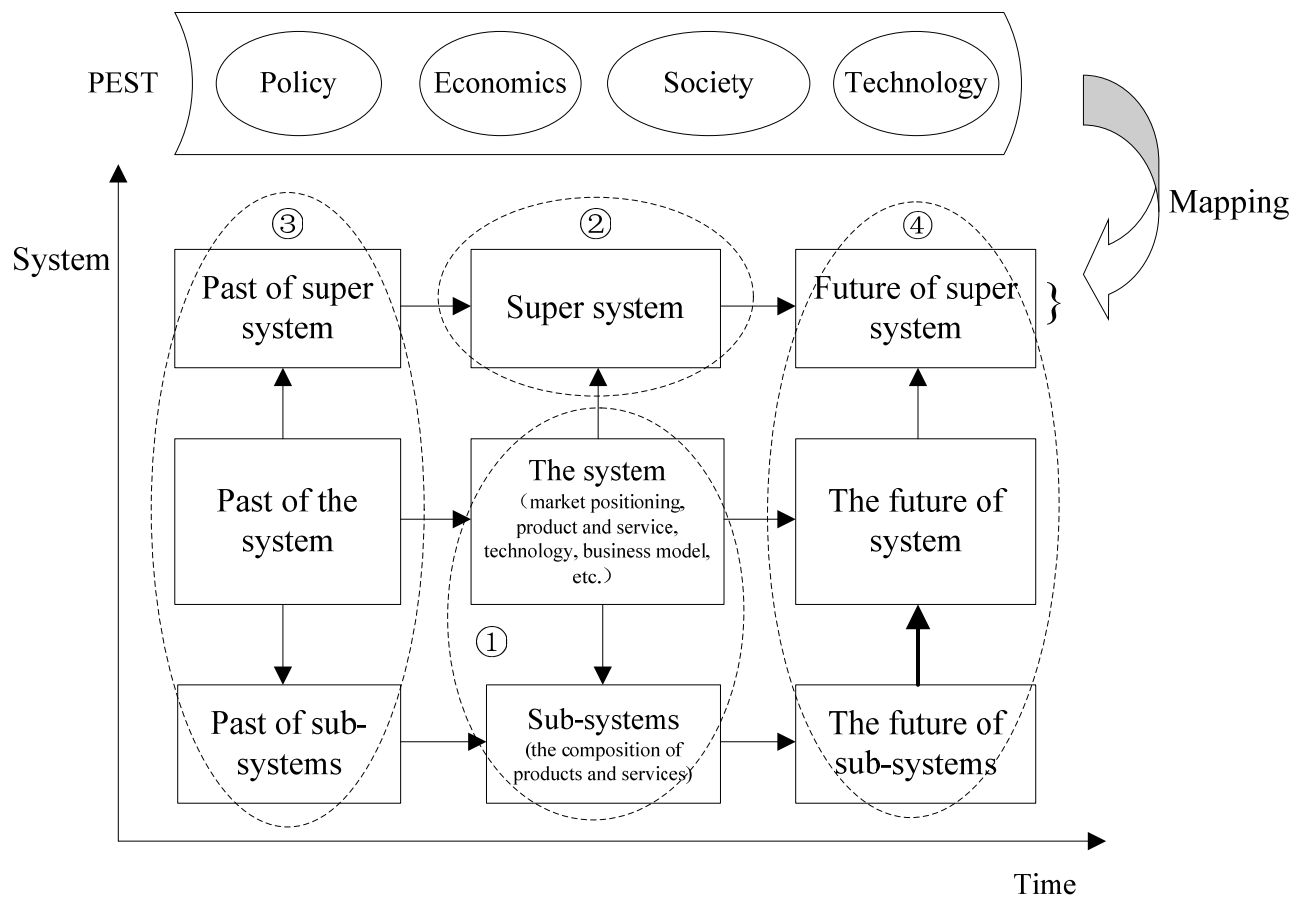

Figure 1. An identification process of entrepreneurship opportunities based on 9 windows and PEST model 


\subsection{The Path of Counseling College Students' Entrepreneurship Plan Project Driven by Effectual Logic}

Effectuation was first proposed by William, and later introduced into the field of management by Sara, who put forward the effect logic theory used to explain the creation of new enterprises and new markets [8]. Effectual logic starts from analyzing the existing means, focusing on "who you are", "what you know" and "who you know"; On this basis, determine what you can do, use a small amount of resources as much as possible to start doing what you can do, and then actively interact with people you know, find possible resource providers and negotiate actual input, so as to obtain the commitment of stakeholders, generate new means or purposes, and realize the continuous expansion of resources [8]. Repeat the above process until a viable start-up appears.

In the process of constructing the project path of mentoring graduate students' entrepreneurship plan, encourage college students' entrepreneurs to use resources from their hands (including who I am? What do I know? Who do I know Start and consider using these resources "what can I do". Then, identify entrepreneurial opportunities based on "9 windows" and PEST and further judge "what can be done". Focus on what can be done, get the commitment of stakeholders and reconstruct new means and purposes through interaction with people you know and meet. Through iteration, constantly update the resources in hand and plan new goals until the resources can support the establishment of new enterprises, the development of new products or the development of new markets.

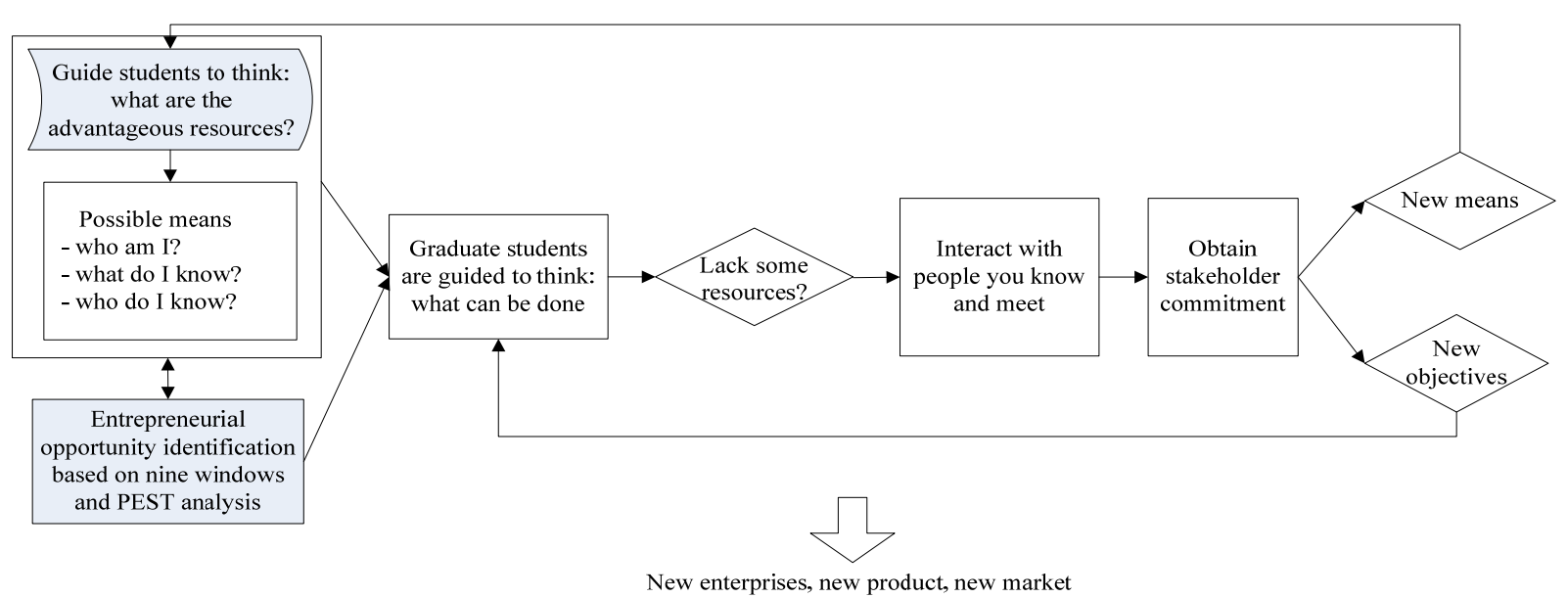

Figure 2. A path of counseling graduate students' entrepreneurship plan project by using 9 windows and PEST under effectual logic

\section{Validation of the Proposed Path}

This study takes the process of tutoring the project team of "Mu Chun", a project aiming to provide low fat and low sugar desserts, to apply for the 2021 graduate students' innovation and entrepreneurship training program as an example, and compares the project planning process by using "entrepreneurship process under the existing effectual logic" and "project path of tutoring graduate students' entrepreneurship program proposed in this study", to test the feasibility and effectiveness of the counseling path proposed in this study.

\subsection{Initial Plan of "Mu Chun" Project by Using the Traditional Effectual Logic}

According to the traditional entrepreneurial process based on effectual logic, the detailed analysis process is as follows:

Step 1: Understand the resources in hand from three aspects: "who I am", "what I know" and "who I know". The project sponsor is familiar with the dessert production process and ingredients, understands the cost of xylitol and the benefits of sugar substitute processing dessert, and knows a friend who runs a dessert shop.

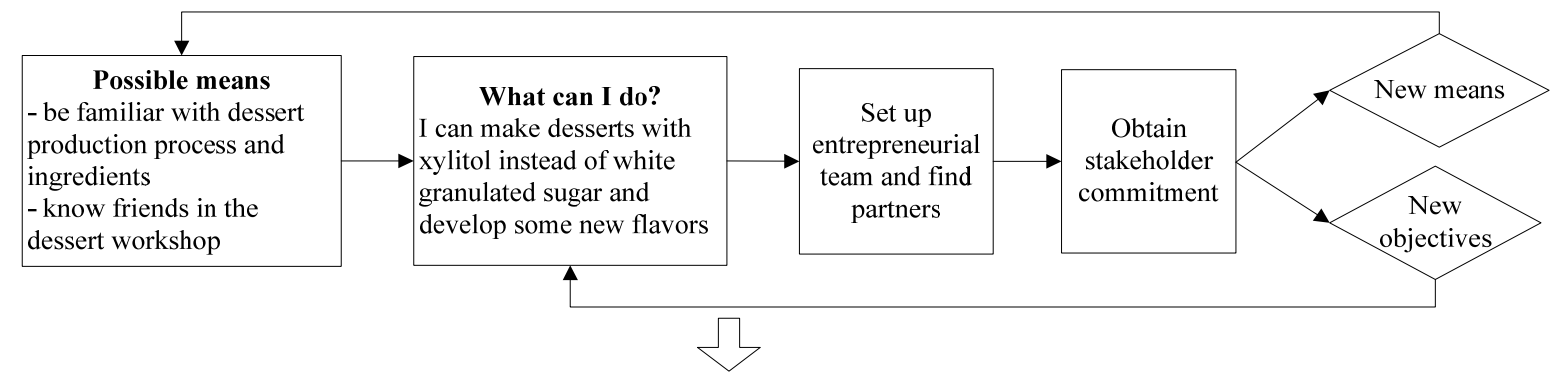

Market positioning is not limited to people with diabetes, plans to increase the target population (including anti cavities, lipid lowering and anti aging groups), and introduce new flavored and new packaged xylitol desserts.

Figure 3. Initial plan of "Mu Chun" project by using the traditional effectual logic 
Step 2: According to the resources in hand, analyze the desserts with xylitol as ingredients and develop some new flavors.

Step 3: Set up an entrepreneurial team and find partners, obtain the commitment of stakeholders, and trial produce a xylitol mung bean cake for market test, which has been highly recognized by the tasters.

Step 4: Create new segments and new purposes, targeting the diabetic population, people with high fat, sugar and hypertension, the children with anti cavities and the group of anti-aging people, and introducing new flavored and new packaged xylitol desserts.

\subsection{New Plan of "Mu Chun" Project By Using the Proposed Path}

As provided in Section 3.2, the analysis process is as follows.

Step 1: It is same as the Step 1 in Section 4.1.

Step 2: Combine the resources in hand, select the low sugar dessert market, and apply the entrepreneurial opportunity identification method based on 9 windows and PEST analysis to develop opportunities. The analysis process is as follows (see Figure 4).

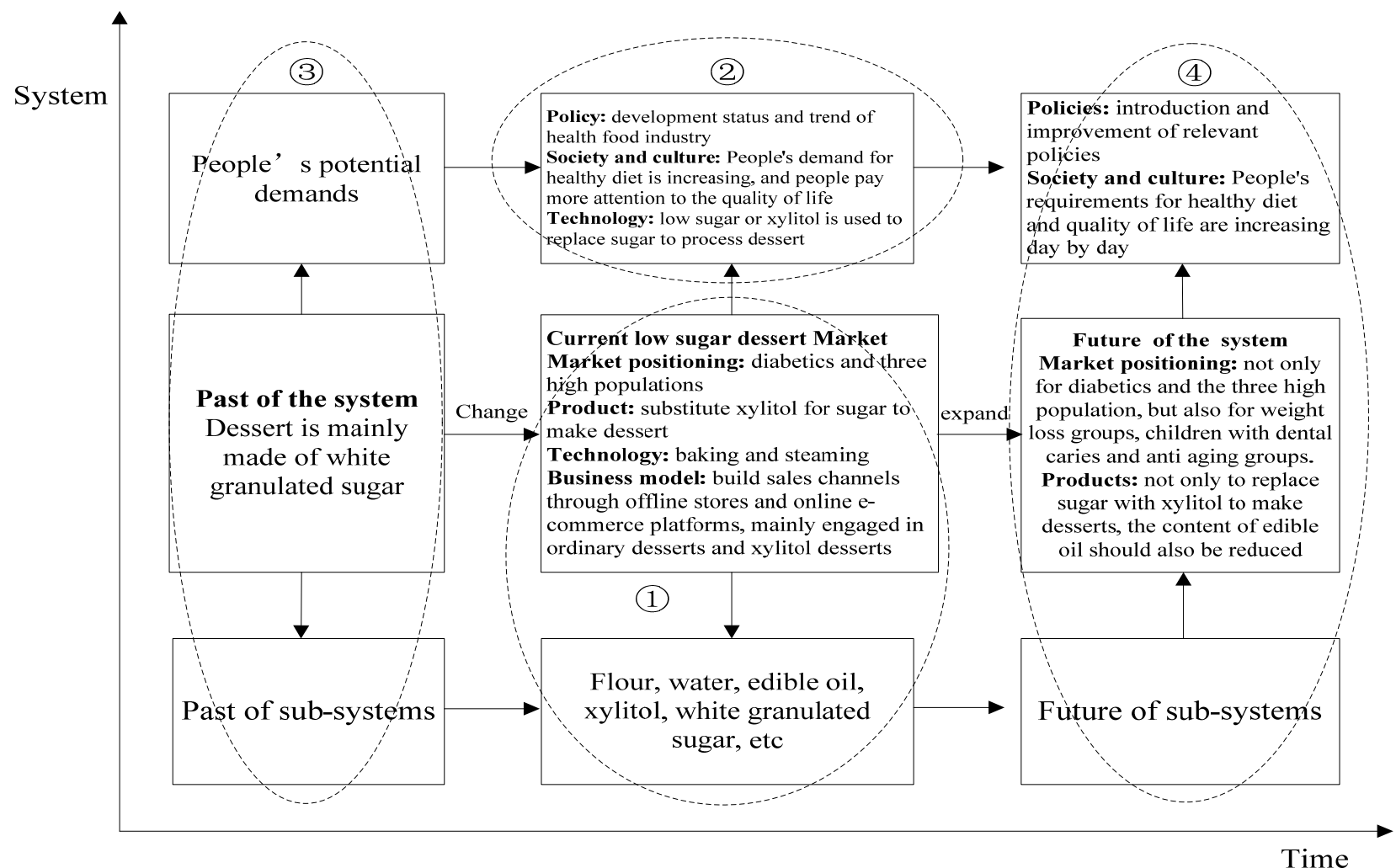

Figure 4. Analysis process of "Mu Chun" project by using the proposed path

(1) Analyze the current system and subsystems. In the system layer, market positioning: diabetes and three high crowd; products and services: mainly engaged in ordinary desserts and xylitol desserts; technology: baking and steaming; business model: build sales channels through offline stores and online e-commerce platforms, and take selling desserts as the main profit source; analyze industry background. Subsystem layer: the ingredients of the product are mainly composed of flour, water, white granulated sugar, xylitol, edible oil, stuffing, etc.

(2) Analyze the "super system" of the current system. "The report on nutrition and chronic diseases of Chinese residents (2020)" shows that the overweight and obesity rate of Chinese adult residents exceeds $50 \%$, the overweight and obesity rate of children and adolescents aged 6 to 17 is close to $20 \%$, and the overweight and obesity rate of children under 6 is $10 \%$ [9]. According to expert analysis, the imbalance of energy intake and energy expenditure is the direct cause of individual overweight and obesity [10]. As people's demand for healthy diet increases, not only are there demand for sugar free desserts in diabetes and the three high population, but also for people with low fat, anti-aging and caries prevention, there are also demands for low sugar and low-fat desserts.

(3) By observing backwards along the time axis and analyzing the past tense of the current system and subsystems and the past tense of the super system, it is found from some subsystems that desserts with white granulated sugar as ingredients from the past to the present. Compared with the changes in the past, there are desserts with xylitol as ingredients, and a variety of flavors and styles have been added. These changes stem from the restriction of sugar intake in some people.

(4) According to the reasons found in the third step, combined with the "super system" resources, analyze whether these reasons can continue to promote the evolution of subsystems. With the increasing demand for healthy diet and quality of life, it will continue to drive the development of sugar free desserts. "The market position of the current system" is not limited to diabetic patients and the people with hyperlipidemia, hyperglycemia and hypertension, but also includes the fat reducing crowd, the anti-aging population and the prevention of dental caries, and will fully integrate the cultural and technological factors into the product design concept. In addition, due to the influence of super system 
resources, it is planned to pay attention to the publicity of the role of sugar alcohol on the body in publicity and promotion, so as to enhance people's understanding and trust in xylitol.

Step 3: Find the missing resources according to the identified entrepreneurial opportunities, establish an entrepreneurial team, seek the commitment of stakeholders, and solve the problems of processing, plant and equipment. The business model of the project is designed as follows:

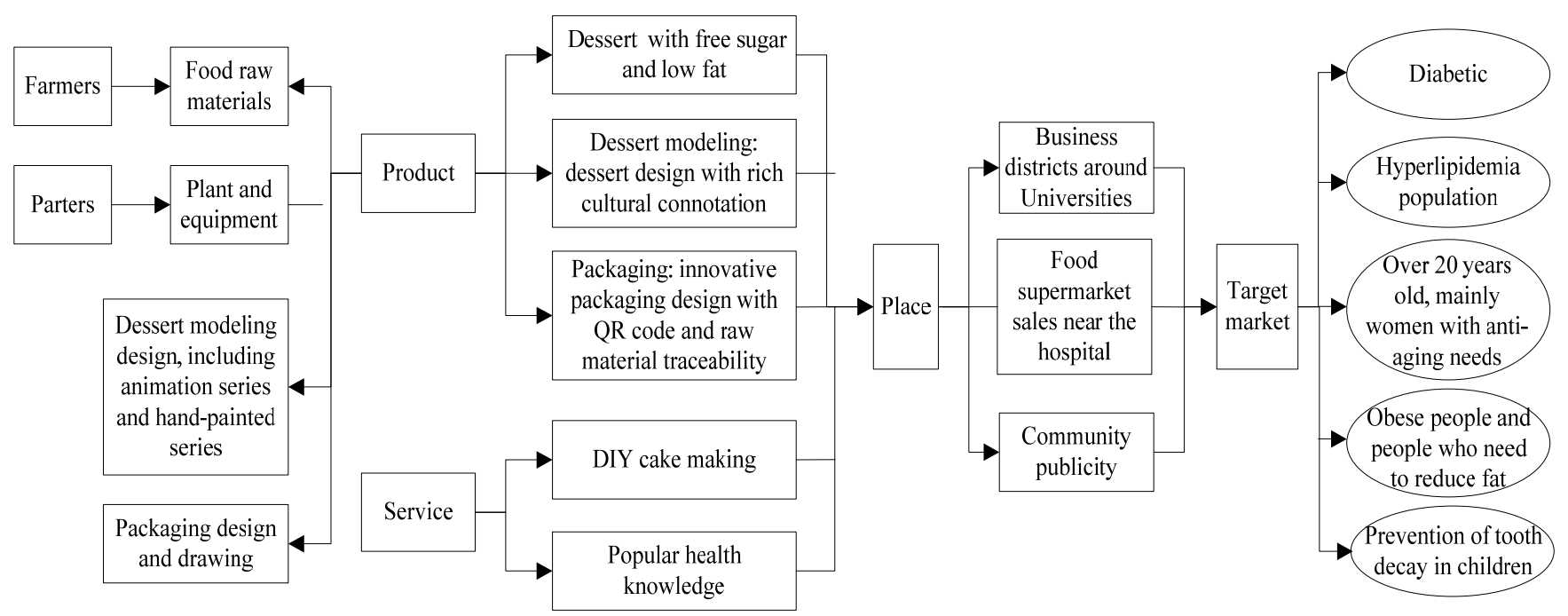

Figure 5. Business model of "Mu Chun" plan

Finally, this "Mu Chun" project won the national project approval in the 2021 graduate students' innovation and entrepreneurship training program. In addition, the undergraduate entrepreneurship plan projects in 2020, which just used the traditional method, are selected as the reference group. In 2021, the proposed method was used to guide the undergraduate students to conduct comprehensive analysis along the time axis and system axis, and analyze the state and causes of the evolution of the system along the time axis, then to develop entrepreneurial opportunities. Similarly, they apply for the graduate students' innovation and entrepreneurship training program. By comparison, in 2020, a total of 6 groups of student teams won the school level project approval. For the projects tutored in the 2021, one won the national level project approval, one won the municipal level project approval, and two won the school level project approval. It can be seen that the project path of counseling graduate students' entrepreneurship plan proposed in this study has strong practicability and application value.

\section{Conclusions}

Aiming at the existing problems in the ability of tutoring and incubating graduate students' innovation and entrepreneurship projects, this study adopts "9 windows" resource analysis method in TRIZ and PEST analysis model, breaks through the thinking inertia and innovation limitations caused by discipline fields, encourages interdisciplinary integration, stimulates students to make full use of resources to generate innovation and identify entrepreneurship opportunities, constructs the ability improvement path of counseling graduate students' innovation and entrepreneurship projects driven by effectual logic. Finally, in the process of counseling graduate students' innovation and entrepreneurship project, the feasibility and effectiveness of this path are tested through comparative analysis.

\section{Acknowledgment}

This study is supported by "2020 Tianjin College Students' innovation and entrepreneurship special project-Research on the ability of counseling and incubation college students' innovation and entrepreneurship project", Project No.: TJZC202001001.

\section{References}

[1] Pei Yaonan, Wang Chengwu, Zhou Jie. Research on Influencing Factors of College Students' innovation and entrepreneurship education driven by project -- Empirical Analysis Based on MOA model [J]. Exploration of higher education, 2019 (7): 108-116 (in Chinese).

[2] Zhang Yuli, Xue Hongzhi, Chen Hansong, Li Huajing. Entrepreneurship management [M]. Beijing: China Machine Press, 2016.06 (in Chinese).

[3] Deng Hanhui. Foundation of entrepreneurship [M]. Beijing: Peking University Press. 2016 (in Chinese).

[4] Yang Hui. On College Students' entrepreneurship guidance methods [J]. Business, 2013,01:272(in Chinese).

[5] Liu Jianchang. Research on personalized and differentiated counseling of entrepreneurship projects in Higher Vocational Colleges [J]. Employment of Chinese college students, 2018,12:55-59(in Chinese).

[6] Tan Runhua. TRIZ and Its Application -- process and method of technological innovation [M]. Beijing: Higher Education Press, 2010(in Chinese).

[7] Zlotin B, Zuaman A. The concept of resources in TRIZ: past, present and future
$[\mathrm{EB} / \mathrm{OL}]$. https://ideationtriz.com/new/materials/finalconceptresources.p df, [2012-9-5].

[8] Read S , Dew N, Sarasvathy S D, et al. Marketing Under Uncertainty: The Logic of an Effectual Approach[J]. Journal of Marketing, 2009, 73(3):1-18.

[9] Report on nutrition and chronic diseases of Chinese Residents. 2020. https://baike.baidu.com/item/report on nutrition and chronic diseases of Chinese residents.

[10] https://xw.qq.com/cmsid/20210416A058VD00. 